\title{
Research on the Shape Precison of the Micro-groove in the Silicon under Diamond Fly-cutting
}

\author{
Yanyan $\mathrm{YAN}^{1, \mathrm{a}}$, Chengyun $\mathrm{YANG}^{1, \mathrm{~b}}$ and Runxing $\mathrm{WANG}^{1, \mathrm{c}}$ \\ ${ }^{1}$ School of Mechanical and Power Engineering, Henan Polytechnic University, Jiaozuo, Henan \\ Province, China \\ ayyy@hpu.edu.cn, b295637683@qq.com,, cwangrunxing2014@163.com
}

\begin{abstract}
Based on the mechanical physical characteristics of the silicon wafers and the superior processing character of the diamond fly-cutting, the diamond fly-cutting was applied to the machining of the microgroove in the silicon, and the effect of the processing parameters on the machining shape precision of the microgroove arrays in the silicon was mainly studied. Firstly, the cutting model of the microgroove in cross section under the diamond fly-cutting was established and the theoretical curvature radius of the microgroove was obtained. Secondly, the shape precision of the microgroove is deduced. Finally, the processing experiment of the microgroove in the silicon was carried out. The experimental results reveal that the higher spindle speed $n$ and the smaller cutting feed $f$ are beneficial to machine the better microgroove in the silicon, but it is important to choose the appropriate cutting depth to machine the better microgroove in the silicon. .
\end{abstract}

\section{Introduction}

Monocrystal silicon combines so many excellent properties (such as the stable chemical properties, the enough mechanical strength and excellent electric properties etc.) that it has been applied widely in the optical communication field and the electromechanical device integration field $[1,2,3]$. Especially, it is of the important significance that the microgroove arrays in the silicon wafer can be machined in the field of optical-communication and the integration on chip. However, it is a kind of hard brittle materials, so it is difficult that the cutting process of silicon is actually a plastic deformation process under the external force, and it is easy that it gets broken, therefore, it becomes a difficult problem in the ultra-precision machining field that the high quality microgroove arrays in the silicon pieces is machined [4].

With the development of the cutting tool materials and the ultra-precision processing equipment, the new idea, which the ultra-precise surface in hard brittle materials is machined not by the grinding method, but by the cutting method, occurs to deal with the contradiction between high efficiency and good surface quality in the ultra-precision machining field, and many scholars begin to work on the problem. Among many studies, diamond fly-cutting becomes a new ultra-precise machining method which can deal with hard brittle materials 
with surface microstructures because it has the superior processing performance, and diamond fly-cutting can deal with the more complex surface microstructures than other machining method of the diamond and similar super-hard cutting tool. And the diamond fly-cutting has an important impact in the microstructure process of linear microgroove, microgroove arrays that are composed of many intersecting lines, repetitive prismatic matrix and pyramidal matrix [5]. In addition, the diamond fly-cutting has very high processing efficiency, which can obtain the microstructure surface with nanoscale or sub-micron scale accuracy without the polishing processing immediately [6]. As a result, the diamond fly-cutting becomes a new ultraprecise machining method in the field of ultra-precise processing of hard brittle material.

In this paper, the microgroove arrays in the silicon were machined by diamond fly-cutting, and the effect of the main the processing parameters on the formation of the microgroove in the silicon was studied. Considering the cutter nose as an arc cutting edge during the cutting process, the cutting model of the microgroove in cross section was firstly built, and the curvature radius of the cutter nose is given, then the shape precision of the microgroove is deduced. Finally, the experimental platform of diamond fly-cutting was built, which consists of vertical machining center (VMC850E), fly-cutting plate, the bracket of work piece and PCD cutting tool, and then the experiment of the microgroove in silicon machined by diamond fly-cutting is carried out on the platform, and the influence of the processing parameters on the shape precision of the silicon microgroove was analyzed, so it is expected that the new research approach is offered for the ultra-precision machining of hard brittle material.

\section{The Analysis of the Effect of the Curvature Radius of the Cutter Nose on the Machined Microgroove by Diamond Fly-cutting}

The diamond fly-cutting is single point diamond turning that is the intermittent process, and Fig. 1 shows the schematic of diamond fly-cutting, and establishing the xoy coordinate is shown as Fig.1. According to Fig.1, the cutter rotates around the central point of fly-cutter disk, meanwhile the workpiece moves along the tangential direction $\mathrm{x}$ of the fly-cutter disk. So the cutting path of the diamond cutter in the workpiece is shown as followed.

$$
\left\{\begin{array}{l}
x=\mathrm{R} \sin (2 \pi \mathrm{n} t)+f t \\
\mathrm{y}=\mathrm{R}+\mathrm{R} \cos (2 \pi \mathrm{n} t)
\end{array}\right.
$$

Where $\mathrm{R}$ is the distance between the cutter tip and the central point of fly-cutter $\operatorname{disk}(\mathrm{mm})$, $n$ is the rotational speed of the spindle $(\mathrm{r} / \mathrm{min}), f$ is the feed of the workpiece $(\mathrm{mm} / \mathrm{min})$, $t$ is the period of time from original state to the current state(min).

According to Eq.1, the simulation results of the cutting path of the fly-cutter in the workpiece are shown in the Fig. 2. According to Fig. 2, the cutting path of the fly-cutter in the workpiece is composed of many ellipses, so the cutting way of diamond fly-cutting is similar to the cutting way of rotating tool, and it is the discontinuous rotary cutting.
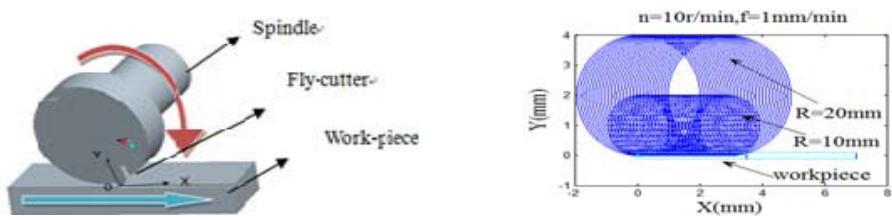
However, the diamond fly-cutting is different from the traditional diamond cutting, and its processing parameters are smaller during the cutting process, so the cutter nose is not considered as an ideal tip during the cutting process, and it is necessary that the curvature radius of the cutter nose is considered during the cutting process. Fig. 3 shows the cutter nose of the PCD cutter used in the experiment. According to Fig.3, the materials is mostly removed by the cutter nose during the diamond fly-cutting, so the size of the cutter nose is an important parameter during the cutting process, and the machined quality of the microgroove in the silicon is mainly affected by the size of the cutter nose. Fig.4 shows the cutting model of the microgroove in cross section. According to Fig.4, the theoretical curvature radius of the microgroove is deduced in Eq.2 on the assumption that the circular profile of the cutter nose is tangent to its cutting edge.

$$
r=a_{\mathrm{p}} / 2+b^{2} /\left(8 a_{p}\right)
$$

Where $r$ is the theoretical curvature radius of the microgroove $(\mathrm{mm}), a_{\mathrm{p}}$ is the depth of the microgroove $(\mathrm{mm}), \mathrm{b}$ is the width of the microgroove $(\mathrm{mm})$.
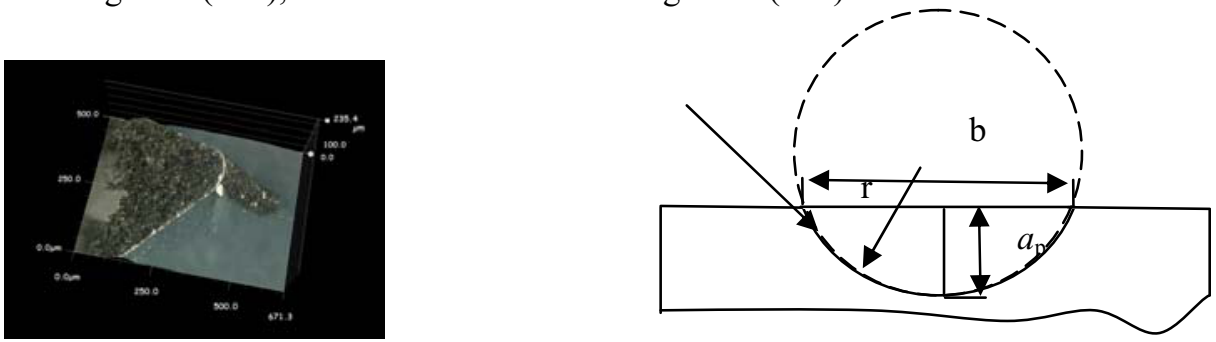

Fig.3 the cutter nose of the PCD cutter Fig.4 the cutting model of the microgroove in cross section

According to Eq.2, there is the nonlinear relationship between the cutting depth and the cutting width of microgroove, and the theoretical curvature radius $r$ of the microgroove can be deduced when its cutting depth and the cutting width are measured. So the roundness error of microgroove can be calculated according to Eq.3

$$
\Delta Z=\left|\left(\mathrm{r}_{\max }+r_{\min }\right) / 2-r\right|=\left|r^{\prime}-r\right|
$$

Where $\Delta Z$ is the roundness error of microgroove, $r_{\max }$ is the radius of minimum circumscribed circle of microgroove profile $(\mathrm{mm}), \mathrm{r}_{\min }$ is the radius of maximum inscribed circle of microgroove profile $(\mathrm{mm}), \mathrm{r}$ is the curvature radius of the microgroove $(\mathrm{mm})$.

\section{The Machining Test of the Microgroove in the Silicon by Diamond Fly-cutting}

\subsection{Test Platform}

The experimental platform of diamond fly-cutting is shown in Fig. 5. It consists of vertical machining center (VMC850E), fly-cutting plate, the bracket of work piece and PCD cutting tool (its front rake angle is $0^{0}$, its relief angle is $5^{0}$, the curvature radius of the tool nose is $0.01 \mathrm{~mm}$ and the angle between the cutting edge is $55^{\circ}$.).

\subsection{Test Conditions}

P-type silicon slice $(\Phi 50 \times 2 \mathrm{~mm})$ is selected as the machining object, and its crystal orientation of growth is $\langle 111>$. The experimental condition is shown in the Tab. 1. The machined surface of microgroove is characterized by the white light interferometer (Talysurf 
$\mathrm{CCI}$ ) and the 3D microscope with super depth field respectively, and the machined workpiece is shown in Fig.6.

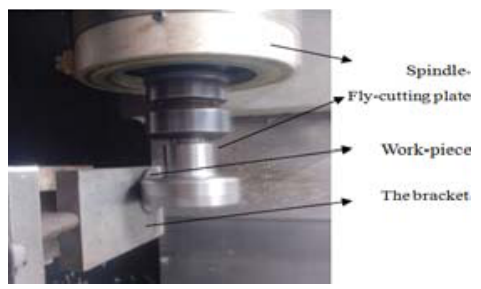

Fig.5 The processing site of diamond fly-cutting

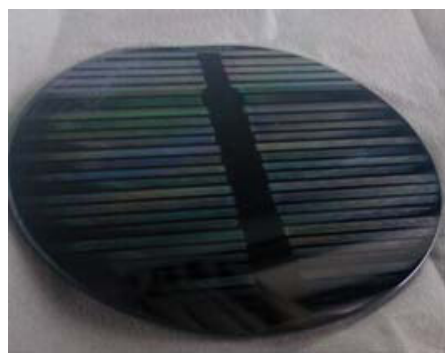

Fig.6 The machined workpiece

\section{Results and Discussion}

Fig.7 shows the profile curve of the silicon microgroove characterized by the white light interferometer (Talysurf CCI). According to Fig.7, the profile of the microgroove in the silicon can be measured, and the height and width of the microgroove in the silicon can be obtained, so the theoretical curvature radius $r$ of the microgroove can be calculated according to Eq.2, the curvature radius of the microgroove $r$ can be measured by the 3D microscope with super depth field, then the roundness error of microgroove $\Delta Z$ can be calculated according to Eq.3, which is used as the index of the shape precision of the microgroove. In addition, the profile of the microgroove in the silicon reveals the formation of silicon under diamond fly-cutting changes from extrusion process to shear slip process, so the microgroove array in the silicon may be machined in the plastic removal mode by diamond fly-cutting when the cutting depth $a_{p}$ is less than the critical cutting depth $a_{p c}$.

Tab.1 The Orthogonal Experiment $\mathrm{L}_{25}\left(5^{3}\right)$

\begin{tabular}{|c|c|c|c|}
\hline Factor & Cutting depth $a_{\mathrm{p}}$ & Workpiece feed $f$ & Spindle speed $n$ \\
\hline Level & $(\mu m)$ & $(\mathrm{mm} / \mathrm{min})$ & $(\mathrm{r} / \mathrm{min})$ \\
\hline 1 & 1 & 1 & 4000 \\
\hline 2 & 2 & 2 & 5000 \\
\hline 3 & 3 & 3 & 6000 \\
\hline 4 & 4 & 4 & 7000 \\
\hline 5 & 5 & 5 & 8000 \\
\hline
\end{tabular}

\subsection{The Influence of the Spindle Speed $n$ on the Shape Precision $\Delta Z$ of the Microgroove in the Silicon}

Fig. 8 shows the influence of the spindle speed $n$ on the shape precision $\Delta Z$ of the microgroove in the silicon. According to Fig.8, the shape precision $\Delta Z$ of the microgroove in the silicon decreases with the spindle speed $n$ obviously when the spindle speed $n$ is within $0 \sim 7000 \mathrm{r} / \mathrm{min}$, but the shape precision $\Delta Z$ of the microgroove in the silicon changes has little change when the spindle speed $n$ is within $7000 \mathrm{r} / \mathrm{min} \sim 8000 \mathrm{r} / \mathrm{min}$. so the higher spindle speed $\mathrm{n}$ is beneficial to machine the microgroove in the silicon. 


\subsection{The Influence of the Cutting Feed $f$ on the Shape Precision $\Delta Z$ of the Microgroove in the Silicon}

Fig.9 shows the relationship between the cutting feed $f$ and the shape precision $\Delta Z$ of the microgroove in the silicon. According to Fig.9, the shape precision $\Delta Z$ of the microgroove in the silicon increases with the cutting feed $f$, and the change of its value is obvious, that is to say, the smaller cutting feed $f$ improves the machining quality of the microgroove in the silicon.

\subsection{The Influence of the Cutting Depth ap on the Shape Precision $\Delta Z$ of the Microgroove in the Silicon}

Fig.10 shows the relationship between the cutting depth $\mathrm{a}_{\mathrm{p}}$ and the shape precision $\Delta Z$ of the microgroove in the silicon. According to Fig.10, the shape precision $\Delta Z$ of the microgroove in the silicon increases with the cutting depth $a_{p}$ when the cutting depth $a_{p}$ is greater than $2 \mu \mathrm{m}$, but the shape precision $\Delta Z$ of the microgroove decreases with the cutting depth $a_{p}$ when the cutting depth $a_{p}$ is less than $2 \mu \mathrm{m}$. The reason is that, it is found that the silicon removal way alters from the plastic removal way to the brittle removal way with the increasing of the cutting depth during the diamond fly-cutting, and there is the plough effect during the plastic removal mode when the cutting depth is less than $2 \mu \mathrm{m}$. ( $f=10 \mathrm{~mm} / \mathrm{min}, n=2000 \mathrm{r} / \mathrm{min}$ )

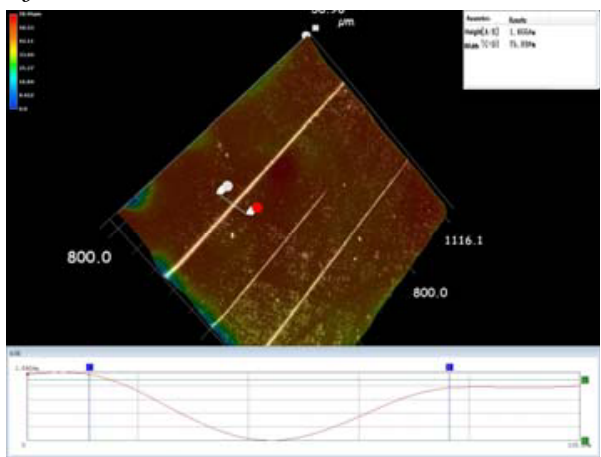

Fig.7 The profile curve of the silicon microgroove

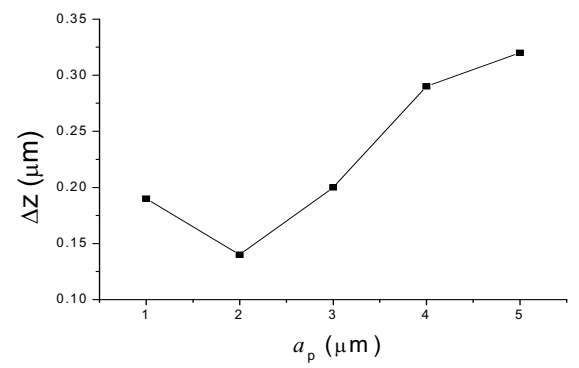

Fig.9 The shape precision $\Delta Z$ under different $f$

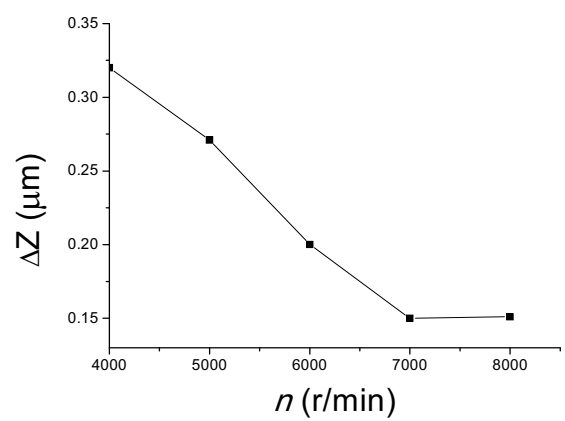

Fig. 8 The shape precision $\Delta Z$ under different $n$

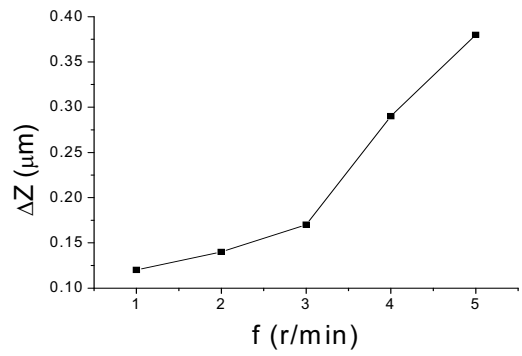

Fig.10 The shape precision $\Delta Z$ under different $\mathrm{a}_{\mathrm{p}}$

\section{Summary}

Based on the theoretical analysis and the experimental discussion, the diamond fly-cutting is suitable for the machining of the microgroove in the silicon, and the effect of 
their processing parameters on the shape precision of the microgroove array in the silicon is summarized as follows.

1. It can be obtained, according to the cutting model of the diamond fly-cutting and analysis of the tool cutting path, that the cutting way of diamond fly-cutting is similar to the cutting way of rotating tool, and it is the discontinuous rotary cutting.

2. During the diamond fly-cutting, it is found that the shape precision $\Delta Z$ of the microgroove in the silicon decreases with the spindle speed $n$ obviously when the spindle speed $n$ is within $0 \sim 7000 \mathrm{r} / \mathrm{min}$, and the shape precision $\Delta Z$ of the microgroove increases with the cutting feed $f$, so the higher spindle speed $n$ and the smaller cutting feed $f$ are beneficial to machine the better microgroove in the silicon.

3. Under diamond fly-cutting, the shape precision $\Delta Z$ of the microgroove in the silicon increases with the cutting depth $a_{p}$ when the cutting depth $a_{p}$ is greater than $2 \mu \mathrm{m}$, but it decreases with the cutting depth $a_{p}$ when the cutting depth $a_{p}$ is less than $2 \mu \mathrm{m}$, so it is important to choose the appropriate cutting depth to machine the better microgroove in the silicon.

\section{Acknowledgement}

This research was sponsored by the National Natural Science Foundation of China (51205112),

\section{References}

1. Y. Li, Fabrication of Si-V groove used for fiber array, J. Infrared and laser engineering, 2002, 31 (5) 447-450.

2. J. Chen, Y. Zhu K, S. Y. Liu, Q. S. Qian, W. F. Sun, Breakdown characteristic of SOI trench structure, J. Journal of Southeast University, 2012, 42 (2)234-238.

3. J. Q. Liang, F. J. Hou, One dimension optical fiber array with silicon V-grooves, J. Opt. Precision Engineering, 2007, 15(1): 89- 94.

4. Q. L. Zhao, S. Dong, Y. Zhao, The research of effects of brittle single crystal material's anisotropy on the diamond turned surface quality, J. China mechanical engineering, 2000, 11 (8) 855-860.

5. Q. L. Zhao, B. Guo, H. Yang, Y. L. Wang, Technological parameter optimization of micro- structured surfaces by diamond fly-cutting, J. Optics and Precision Engineering, 2009, 17(10) 2512-2519.

6. M. A. Davies, C J. Evans, S. R. Patterson, Application of precision diamond machining to the manufacture of microphotonics components, J. SPIE, 2003. 